\author{
S. P. Lemburg' ${ }^{1}$ \\ T. Kagel ${ }^{1}$ \\ S. Grootonk ${ }^{2}$ \\ S. Ewig ${ }^{3}$ \\ T. T. Bauer ${ }^{2}$ \\ G. Schultze-Werninghaus ${ }^{2}$ \\ V. Nicolas ${ }^{1}$ \\ C. M. Heyer ${ }^{1}$
}

\section{Stent-Implantationsplanung bei tumor-assoziierter, brochoskopisch nicht passierbarer Trachealstenose - Eine Indikation für die virtuelle CT-Bronchoskopie}

Non-passable Tumor-associated Stenosis of Trachea and Stent-implantation an Indication for Virtual CT-Bronchoscopy

\section{Zusammenfassung}

Wir berichten über einen 50-jährigen Patienten mit bekanntem nicht-kleinzelligem Lungentumor, bei dem im Vorfeld eine tumorbedingte, rechtsseitige Hauptbronchusstenose mittels Neodym-YAG-Laser behandelt wurde. Im Verlauf entwickelte der Patient eine Totalatelektase der rechten Lunge und eine den linken Hauptbronchus einbeziehende Stenose der distalen Trachea. Eine vollständige Visualisierung dieser langstreckigen Stenose durch die konventionelle Bronchoskopie gelang nicht. Die Multidetektor-CT-generierte virtuelle Tracheobronchoskopie zeigte die vollständige Ausdehnung des Befundes und ermöglichte zudem eine exakte prä-interventionelle Planung vor Stent-Implantation. Im Rahmen einer Kontrolluntersuchung ließen sich wiederum mit Hilfe der virtuellen 3D-Tracheobronchoskopie die korrekte Lage des Stents und seine Durchgängigkeit verifizieren. Unser Fallbericht beschreibt eine sinnvolle, schnell verfügbare und nicht-invasive Methode, das Tracheobronchialsystem bei Patienten mit intraluminalem Tumorwachstum adäquat darzustellen und so vor und nach Stent-Implantation zu evaluieren.

\section{Abstract}

We report about a male 50-year-old patient with known nonsmall cell lung cancer and tumor-associated stenosis of the right main bronchus already treated with Neodym-YAG-laser coagulation. Eight months later the patient was readmitted to hospital and revealed complete occlusion of the right main bronchus and subtotal stenosis of the distal trachea and the left main bronchus. Conventional tracheobronchoscopy failed to visualize the post-stenotic parts of the left main bronchus due to the length and degree of stenosis. Multidetector-CT-generated virtual tracheobronchoscopy was able to demonstrate complete intraluminal tumor extent thus making precise pre-interventional measurements for stent implantation possible. Furthermore, postinterventional re-evaluation by virtual tracheobronchoscopy confirmed correct position and patency of the stent located within the distal trachea and the left main bronchus. Our case report demonstrates a non-invasive and easy approach for evaluating the tracheobronchial system in a patient with tumor-associated airway stenosis including the possibility for viewing beyond poststenotic segments. genossenschaftliche Kliniken Bergmannsheil, Klinikum der Ruhr-Universität Bochum

${ }^{2}$ Medizinische Klinik III, Pneumologie, Allergologie und Schlaf- und Beatmungsmedizin, Berufsgenossenschaftliche Kliniken Bergmannsheil, Klinikum der Ruhr-Universität Bochum

${ }^{3}$ Abteilung für Pneumologie, Infektiologie und Beatmungsmedizin, Augusta-Krankenanstalt, Bochum

Korrespondenzadresse

Dr. Stefan Peter Lemburg · Institut für Diagnostische Radiologie · Interventionelle Radiologie und Nuklearmedizin, Berufsgenossenschaftliche Kliniken Bergmannsheil · Klinikum der Ruhr-Universität Bochum · Bürkle-de-la-Camp-Platz 1 444789 Bochum . E-mail: stefan.lemburg@ruhr-uni-bochum.de

Eingang: 19. Januar 2005 - Nach Revision akzeptiert: 20. Juni 2005 


\section{Einführung}

Die Computertomographie hat in der Diagnostik und Therapie der malignen Lungentumoren einen festen Stellenwert. Die Multidetektor-Computertomographie (MDCT) ermöglicht die Anfertigung annähernd isotroper Datensätze und so eine dreidimensionale Darstellung des erfassten Volumens. Damit sind nichtinvasive, virtuelle Darstellung von Hohlraumsystemen des Körperinneren (Tracheobronchialsystem, Darm) durchführbar. Die Abbildung der Lumina des tracheobronchialen Systems gelingt mit Hilfe der virtuellen MDCT-Tracheobronchoskopie bis auf das Niveau der subsegmentalen Bronchien. Der Stellenwert dieser virtuellen Diagnostik ist vor dem Hintergrund der fehlenden Möglichkeit einer Schleimhautbeurteilung bzw. einer BiopsieEntnahme zum jetzigen Zeitpunkt noch umstritten. Prospektive Studien zur Validierung fehlen, erste klinische Erfahrungen lassen jedoch vermuten, dass die Methode bei intraluminal wachsenden Tumoren oder bei der Planung interventioneller Verfahren wichtige Zusatzinformationen liefern kann.

Wir berichten über einen Patienten mit zentral wachsendem Plattenepithelkarzinom, der sich mit Hämoptysen und progredienter Ruhedyspnoe in unserer Klinik vorstellte. Bereits im Vorfeld war eine tumorbedingte Stenose der Trachea diagnostiziert und behandelt worden. In der jetzt durchgeführten konventionellen Bronchoskopie ergab sich abermals der Nachweis einer ausgedehnten trachealen Stenosierung, welche nicht mit dem Instrument passiert werden konnte. Zur Darstellung der poststenotischen Bronchusverhältnisse sowie zur prä-interventionellen Planung vor Stent-Implantation erfolgte eine MDCT-generierte virtuelle 3D-Tracheobronchoskopie.

\section{Fallbericht}

Ein 50-jähriger Patient wurde initial mit Husten, Dyspnoe, Auswurf, rezidivierenden Fieberschüben, Nachtschweiß und Gewichtsverlust in unserer Klinik stationär aufgenommen. Die durchgeführte Computertomographie zeigte einen rechts-zentral gelegenen Lungentumor mit intraluminalem Wachstum im rechten Hauptbronchus, konsekutiven Teilatelektasen des Mittel- und Unterlappens sowie Infiltration der rechten Pulmonalarterie. Bronchoskopisch bestätigte sich der Verschluss des rechten Hauptbronchus, während histologisch die Diagnose eines verhornenden, hochdifferenzierten Plattenepithel-Karzinoms (Stadium $\mathrm{pT}_{4} \mathrm{G}_{1} \mathrm{~N}_{3} \mathrm{M}_{\mathrm{x}}$ ) gesichert werden konnte. Mittels starrer Bronchoskopie mit Neodym-YAG-Laser-Koagulation gelang zunächst eine suffiziente Rekanalisation der Hauptbronchusstenose. Im Verlauf wurde eine palliative Radiatio appliziert.

Acht Monate später stellte sich der Patient erneut mit Hämoptysen und progredienter Ruhedyspnoe in unserer Klinik vor. Bei laborchemisch erhöhten Entzündungswerten und respiratorischer Partialinsuffizienz $\left(\mathrm{PaO}_{2}\right.$ 54,1 Torr, $\mathrm{PaCO}_{2} 38$,6 Torr, $\mathrm{pH} 7,47$ ) zeigte die Lungenfunktionsanalyse eine mittelgradige obstruktive und restriktive Ventilationsstörung mit erhaltener Teilreversibilität nach Bronchospasmolyse $\left[\mathrm{FEV}_{1} 1,491\right.$ (38,7\% vom Soll), VC $2,351$ ( $48,7 \%$ vom Soll $)]$.

In der Thoraxübersichtsaufnahme fielen ein Abbruch des rechten Hauptbronchus und eine Verengung des linken Hauptbronchus knapp distal der Carina auf. Bei regelrechter Belüftung der linken Lunge zeigte sich zudem eine Minderbelüftung der volumenreduzierten rechten Lunge mit begleitendem Pleuraerguss. Der aus den Voruntersuchungen bekannte rechts-hiläre Tumor war nur angedeutet erkennbar (Abb.1a). Zur weiteren Therapieplanung erfolgte die Anfertigung einer MDCT in Atemanhaltetechnik nach Kontrastmittelgabe (SOMATOM Volume Zoom ${ }^{\circledR}$, Siemens, Erlangen) mit folgenden Parametern: Kollimation $4 \times 2,5 \mathrm{~mm}, 120 \mathrm{kV}, 100 \mathrm{mAs}$, Rekonstruktion axialer Schichten mit 3 und $6 \mathrm{~mm}$ Schichtdicke. Aus dem so gewonnenen Datensatz wurde eine virtuelle 3D-Bronchoskopie mittels Surface-Rendering berechnet (LEONARDO ${ }^{\circledR}$ Workstation, Siemens, Erlangen).

Die MDCT zeigte den großen, inhomogen perfundierten und zentral nekrotischen Tumor rechts-hilär, welcher den rechten Hauptbronchus vollständig okkludierte und zudem in die distale Trachea und über die Bifurkation in den linken Hauptbronchus einwuchs. Im Gegensatz zur Thoraxübersichtsaufnahme fand sich weiterhin eine Überblähung der rechten Lungen im Sinne eines Ventilmechanismus (Abb. 1b u. c u. 2a).

Konventionell-bronchoskopisch konnte vorab ein Einbruch des Tumors in die Trachea mit subtotaler Verlegung des Lumens sowie ausgedehnter Blutung aus dem Tumor dokumentiert werden (Abb. 2b). Eine Passage des Instrumentes jenseits der Stenose war konventionell-bronchoskopisch nicht möglich.

In der virtuellen Bronchoskopie zeigte sich nun - korrespondierend zur konventionellen Bronchoskopie - die durch den Tumor bedingte, vollständige Verlegung des rechten Hauptbronchus sowie eine deutliche Stenose der distalen Trachea und des linken Hauptbronchus (Abb. 2c u. d). Jenseits dieser Stenose konnten die Segment- und Subsegmentbronchien der linken Lunge virtu-

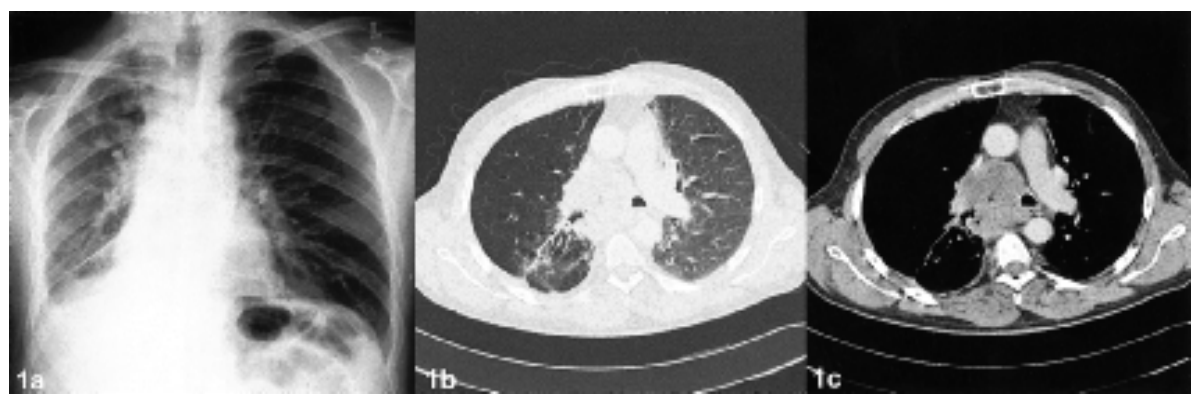

Abb. 1 a) Prä-interventionelle Thoraxaufnahme: Rechtsseitiger Pleuraerguss mit Dystelektasen der rechten Lunge und Mediastinalverlagerung. b) und c) Thorax-CT: Ausgedehnte rechts-hiläre/mediastinale Raumforderung mit subtotalem Verschluss des rechten Hauptbronchus und Überblähung der rechten Lunge durch Ventilmechanismus. 


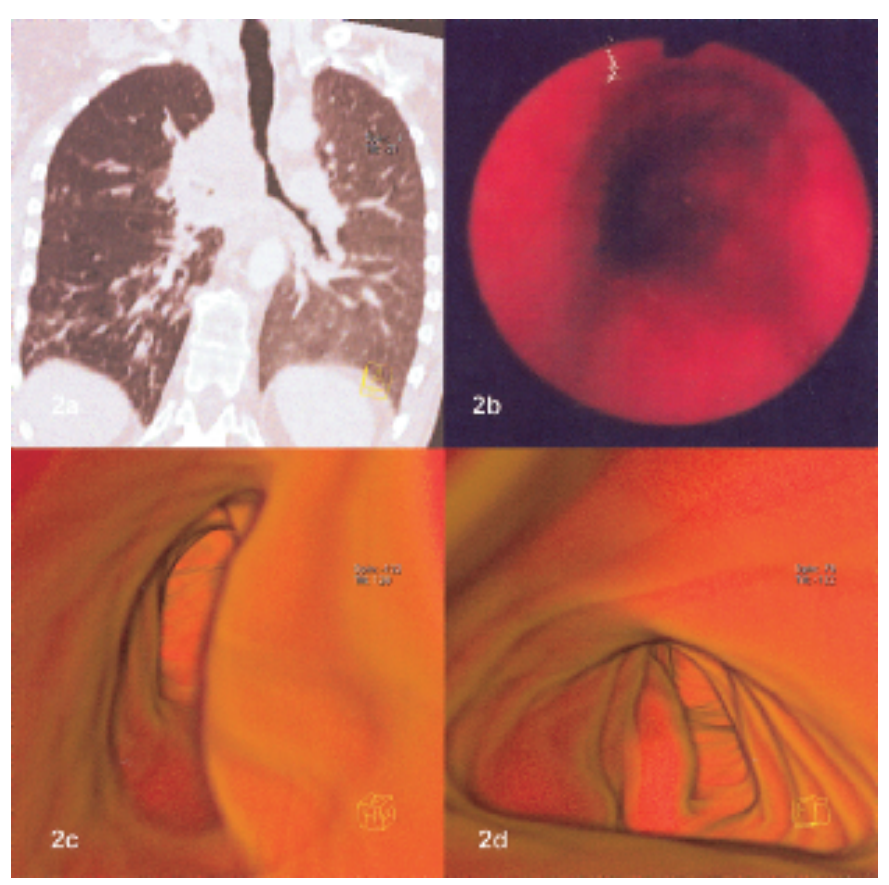

Abb. 2 a) Thorax-CT, coronare Rekonstruktion: Subtotaler Verschluss des rechten Hauptbronchus sowie langstreckige, tumorbedingte Stenose der distalen Trachea und des linken Hauptbronchus. b) Konventionelle Bronchoskopie: Trachealer Tumoreinbruch mit Hämorrhagien und nicht passierbarer Stenose. c) u. d) Virtuelle Tracheobronchoskopie: Subtotale, tumorassoziierte Trachealstenose mit poststenotisch erhaltener Einsehbarkeit der Segmentbronchien.

ell eingesehen werden. Es wurde der Entschluss zur Implantation eines Stents zur palliativen Sicherung der linksseitigen Lungenbelüftung gefasst. Anhand der coronaren Schichten und der virtuellen Endoskopie wurde der linke Hauptbronchus vermessen und die Größe des geplanten Stents bestimmt. Dessen Implantation in die distale Trachea und den linken Hauptbronchus gelang im Anschluss problemlos. Die unmittelbar hiernach angefertigte Thoraxaufnahme zeigte den regelrecht einliegenden Stent mit vollständiger Aufhebung der bronchialen Stenose und wieder unauffälliger Pneumatisation der linken Lunge (Abb. 3a).

Wenige Tage später stellte sich der Patient mit neuerlicher Luftnot in der Klinik vor. Die im Anschluss durchgeführte MDCT mit virtueller Tracheobronchoskopie zeigte den regelrecht einliegenden, durchgängig offenen Stent, welcher die distalen Tumoranteile abdeckte. Zudem fand sich jetzt eine Totalatelektase der rechten Lungen mit konsekutiver Mediastinalverlagerung (Abb. 3b - d).

\section{Diskussion}

Maligne Lungentumoren stellen mit einer Inzidenz von 75/100 000 für Männer und 30/100 000 für Frauen eine der häufigsten tumorbedingten Todesursachen dar. Ca. 90\% der Patienten versterben an ihrer Erkrankung. Der Altersgipfel der Erkrankung liegt bei 60 Jahren mit ansteigender Tendenz ab dem 25 . Lebensjahr [1]. Plattenepithelkarzinome stellen mit einem Anteil von $40 \%$ die größte Gruppe der epithelialen malignen Lungentumoren [1,2]. Die Diagnose eines malignen Lungentumors gelingt oft erst spät; langjähriger Nikotinabusus und therapieresistente pulmonale Infektionen, Husten, Hämoptysen, Gewichtsverlust

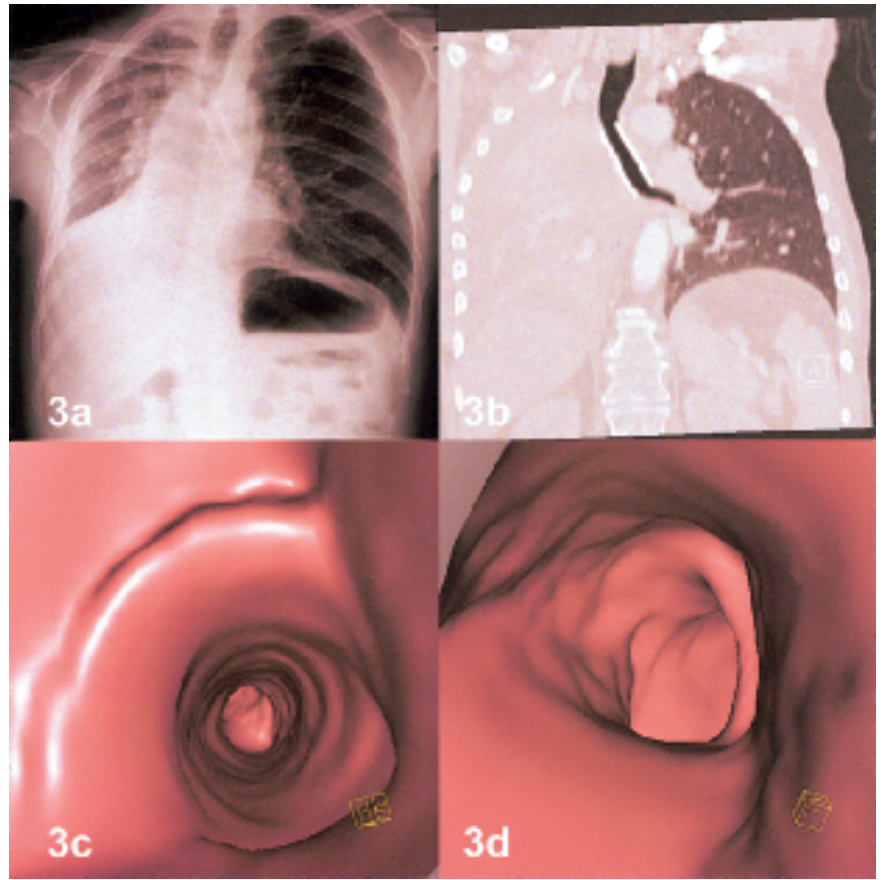

Abb. 3 a) Thoraxübersichtsaufnahme nach Stent-Implantation: Korrekte Stentlage mit regelrechter Belüftung der linken Lunge. b)-d) MDCT mit virtueller Tracheobronchoskopie: Korrekte Stent-Position mit freier Durchgängigkeit des Stents und regelrechter Lungenbelüftung links; jetzt zudem Totalatelektase der rechten Lunge.

und Lymphknotenschwellungen sind richtungsweisend, jedoch nicht obligatorisch. Bei unserem Patienten lagen die Symptome bereits vor; die Bildgebung erbrachte den Nachweis eines fortgeschrittenen Tumorstadiums mit Ummauerung des rechten Hauptbronchus. Verlegungen der zentralen Atemwege sind eine gefürchtete Komplikation bei neoplastischen Prozessen im Mediastinum. Eine effektive palliative Therapieoption besteht wie auch in unserem Fall - in der Laserkoagulation der endoluminalen Tumoranteile mittels starrer Bronchoskopie. Um die längerfristige Offenheit der Atemwege zu gewährleisten, kann zudem ein intraluminaler Stent appliziert werden.

Die Computertomographie hat in der Diagnostik der malignen Lungentumoren ihren festen Stellenwert. Dieses durch Hounsfield 1974 eingeführte Verfahren wurde in den letzten Jahrzehnten kontinuierlich weiterentwickelt. Bei gleichzeitiger Reduktion der Strahlendosis wurden mit Einführung der Spiraltechnik insbesondere durch die Beschleunigung des Scanvorgangs und die lückenlose Abbildung der Bildregion eine Verbesserung der BildAuflösung/-Schärfe und eine Reduktion von Atemartefakten erreicht. Die Einführung der MDCT wurde 1992 mit den ersten 2-Zeiler-Scannern verwirklicht. Heute steht mit den modernen 16-Zeilen-Geräten eine Generation von Scannern zur Verfügung, die über die Akquisition annähernd isotroper Datensätze die Berechnung einer virtuellen 3D-Tracheobronchoskopie erlaubt. Insbesondere die weiter erhöhten Scangeschwindigkeiten der MDCT erlaubt eine Volumenakquisition ohne Kompromittierung durch Atmungs- oder Bewegungsartefakte. Dieses ist insofern von großer Bedeutung, als dass hierdurch tracheobronchiale Stenosierungen vortäuscht werden können [3]. Die dreidimensionale Darstellung der Luftwege ermöglicht eine für den Kliniker 
leicht auf die konventionelle Bronchoskopie übertragbare Darstellung von in den axialen Schichten zweidimensional nachweisbaren Befunden. Auch unter Berücksichtigung der Tatsache, dass prinzipiell alle Bildinformationen bereits in den axialen Schichten enthalten sind, bietet die virtuelle 3D-MDCT-Tracheobronchoskopie gerade bei geplanten Interventionen an den intrathorakalen Luftwegen prinzipiell den Vorteil einer nicht-invasiven, leicht verständlichen räumlichen Darstellung derselben [4]. Bislang fehlt jedoch eine prospektive Evaluation der Methode. Ihre klinische Wertigkeit ist zudem angesichts der fehlenden Möglichkeiten, Schleimhäute und Sekret zu beurteilen bzw. Biopsien durchzuführen, umstritten. Daneben bietet die Methode keine zuverlässigen Aussagen zur Beurteilung funktioneller Prozesse (z.B. Veränderungen der Atemwege innerhalb des Atemzyklus). Schließlich existieren bislang keine allgemein akzeptierten Richtwerte bezüglich der Darstellungskriterien (Dichtefenster/-Weite, Farbgebung, Oberflächendarstellung/-bearbeitung, Kennzeichnung von Modifikationen etc.) [3]. Direkte Rückschlüsse aus der virtuellen Darstellung der Schleimhäute auf die Präsentation in der konventionellen Bronchoskopie sind daher nicht ohne weiteres möglich [5].

Trotz dieser unumstrittenen Nachteile kann die virtuelle Tracheobronchoskopie hinsichtlich der nicht-invasiven Darstellung der Atemwege bei ausgewählten Patienten Vorteile bieten. So ist bei konventionell nicht-passierbaren Stenosen der Atemwege und geplanter Intervention oft ein „Blick“ distal der Stenose möglich. Auf konventionellem Wege sind höhergradige Stenosen in Einzelfällen mit Mini-Bronchoskopen passierbar. Die Darstellung von poststenotischen Bronchusabschnitte kann zudem mittels fokaler Bronchographie unter konventioneller Durchleuchtung durchgeführt werden, wobei anzumerken ist, dass diese zwar kostengünstige Methode hinsichtlich der Beurteilbarkeit poststenotischer Atemwege nur eingeschränkte Informationen liefert. Des Weiteren sind Atemwegsstenosen mittels Laservermessung darstell- und quantifizierbar. Die anhand der MDCT gewonnenen und dreidimensional dargestellten Daten ermöglichen eine Abschätzung des Stenosegrades und somit eine exakte Berechnung der Stentdimensionen prä-interventionell [6,7]. Erste Erfahrungen mit der Methode zeigen weiterhin eine hinreichend genaue Darstellung endoluminaler tumoröser Prozesse [6, 8 - 11]. Ferreti u. Mitarb. demonstrierten an 25 Patienten mit insgesamt 28 endobronchialen Stents eine gute Übereinstimmung zwischen virtueller und konventioneller Bronchoskopie in der postinterventionellen Evaluation [12]. Alternativen zur Behandlung tumor-assoziierter Bronchialstenosen bestehen in der Elektrokoagulation mit dem Argon-Plasma-Laser bzw. in einer Tumorabtragung mittels Neodym-YAG-Laser. Beide Verfahren dienen der Reduktion intraluminaler Tumormassen; bei signifikanter Kompression der Atemwege muss ggf. zusätzlich dilatiert und das Ergebnis mittels Stent-Implantation gesichert werden.

Der vorliegende Fall demonstriert somit eine typische Indikation zur Anfertigung einer virtuellen 3D-Bronchoskopie: Eine konventionell-bronchoskopisch nicht passierbare Atemwegsstenose $[9,13,14]$. Bei unserem Patienten konnten mit Hilfe der virtuellen Bronchoskopie auch die Atemwege distal der nachgewiesenen Stenose des linken Hauptbronchus beurteilt werden. Daneben war auch eine „Inversion“ des virtuellen Blickwinkels möglich, so dass die distale intraluminale Tumorausdehnung visualisiert und quantitativ abgeschätzt werden konnte. Zur Erleichterung der Auswahl eines passenden Stents wurden zusätzlich coronare Schichten berechnet; an ihnen erfolgte die exakte Abmessung der Gesamtdistanz der Stenose, da im Gegensatz zur virtuellen Endoskopie Verzerrungseffekte minimiert werden können $[3,15]$. In Zusammenschau aller Befunde wurde schließlich eine optimale Interventionsplanung möglich. Zugleich demonstriert das Bildmaterial die exzellente Übereinstimmung zwischen konventionell-bronchoskopischem Befund und der virtuellen Darstellung des Tumors, welche auch in anderen Fällen gelang [16].

Zusammenfassend illustriert unser Fallbericht eine sinnvolle Indikation zum Einsatz der virtuellen MDCT-generierten 3D-Tracheobronchoskopie bei Patienten mit konventionell-bronchoskopisch nicht passierbaren, tumor-assoziierten Atemwegsstenosen und deren Einsatz bei geplanter Intervention (Stent-Implantation).

\section{Literatur}

${ }^{1}$ Schmoll HJ, Höffken P, Possinger K. Kompendium Internistische Onkologie 3. Auflage. 1999 Berlin, Heidelberg: Springer-Verlag,

${ }^{2}$ World Health Organization. Histological Typing of Lung Tumors. 2nd Edition. Geneva: World Health Organization, 1981

${ }^{3}$ Summers RM, Feng DH, Holland SM et al. Virtual Bronchoscopy: Segmentation Method for Real-time Display. Radiology 1996; 200: $857-862$

${ }^{4}$ Ferretti GR, Knoplioch J, Bricault I et al. Central Airway Stenoses: Preliminary Results of Spiral-CT-generated Virtual Bronchoscopy Simulations in 29 Patients. Eur Radiol 1997; 7 (6): 854-859

${ }^{5}$ Vining DJ, Liu K, Choplin RH et al. Virtual Bronchoscopy: Relationships of Virtual Reality Endobronchial Simulations to Actual Bronchoscopic Findings. Chest 1996; 109: 549-553

${ }^{6}$ Hoppe H, Beat W, Sonnenschein M et al. Multidetector CT Virtual Bronchoscopy to Grade Tracheobronchial Stenosis. AJR 2002; 178: $1195-1200$

${ }^{7}$ Hoppe H, Dinkel HP, Thoeny H et al. Virtuelle Endoskopie der oberen, zentralen und peripheren Atemwege mit Mehrzeilen-Spiral-CT. Radiologe 2002; 42 (9): 703 -711

${ }^{8}$ Finkelstein SE, Summers RM, Nguyen DM et al. Virtual Bronchoscopy for Evaluation of Malignant Tumors of the Thorax. J Thorac Cardiovasc Surg 2002 May; 123 (5): 967 - 972

${ }^{9}$ Rapp-Bernhardt U, Welte T, Doehring W et al. Diagnostic Potential of Virtual Bronchoscopy: Advantages in Comparison with Axial CT Slices, MPR and MIP? Eur Radiol 2000; 10 (6): 981 - 988

10 Seeman MD, Claussen CD. Hybrid 3D Visualization of the Chest and Virtual Endoscopy of the Tracheobronchial System: Possibilities and Limitations of Clinical Application. Lung Cancer 2001; 32 (3): $237-246$

${ }^{11}$ Burke AJ, Vining DJ, McGuirt WF Jr et al. Evaluation of Airway Obstruction Using Virtual Endoscopy. Laryngoscope 2000; 110 (1): 23 - 29

${ }^{12}$ Ferretti GR, Kocier M, Calaque O et al. Follow-up After Stent Insertion in the Tracheobronchial Tree: Role of Helical Computed Tomography in Comparison With Fiberoptic Bronchoscopy. Eur Radiol 2003; 13 (5): $1172-1178$

${ }^{13}$ McAdams HP, Palmer SM, Erasmus JJ et al. Bronchial Anastomotic Complications in Lung Transplant Recipients: Virtual Bronchoscopy for Non-invasive Assessment. Radiology 1998; 209: 689-695

${ }^{14}$ Fleiter T, Merkle EM, Aschoff AJ et al. Comparison of Real-time Virtual and Fiberoptic Bronchoscopy in Patients With Bronchial Carcinomas: Opportunities and Limitations. AJR 1997; 169: 1591 - 1595

${ }^{15}$ Quint LE, Whyte RI, Kazerooni EA et al. Stenosis of the Central Airways: Evaluation by Using Helical CT With Multiplanar Reconstructions. Radiology 1995; 194: $871-877$

${ }^{16}$ Heyer CM, Bauer TT, Orth M et al. Virtuelle Mehrzeilen-CT-Bronchoskopie in der Diagnostik endobronchialer Tumoren: Fallbericht eines Karzinoid-Tumors. Pneumologie 2003; 57: 272-277 